\section{Actualización en el diagnóstico y manejo del daño hepático agudo grave en el embarazo}

\author{
ALVARO SEPULVEDA-MARTINEZ1', CARLOS ROMERO'2, \\ GUIDO JUAREZ ${ }^{1}$, JORGE HASBUN ${ }^{1}$, MAURO PARRA-CORDERO
}

\section{Causes and management of severe acute liver damage during pregnancy}

Abnormalities in liver function tests appear in 3\% of pregnancies. Severe acute liver damage can be an exclusive condition of pregnancy (dependent or independent of pre-eclampsia) or a concomitant disease. HELLP syndrome and acute fatty liver of pregnancy are the most severe liver diseases associated with pregnancy. Both appear during the third trimester and have a similar clinical presentation. Acute fatty liver may be associated with hypoglycemia and HELLP syndrome is closely linked with pre-eclampsia. Among concomitant conditions, fulminant acute hepatitis caused by medications or virus is the most severe disease. Its clinical presentation may be hyper-acute with neurological involvement and severe coagulation disorders. It has a high mortality and patients should be transplanted. Fulminant hepatic failure caused by acetaminophen overdose can be managed with n-acetyl cysteine. Because of the high fetal mortality rate, the gestational age at diagnosis is crucial.

(Rev Med Chile 2015; 143: 627-636)

Key words: Acetaminophen; Fatty liver; HELLP; Liver failure; Pregnancy complications; Syndrome.

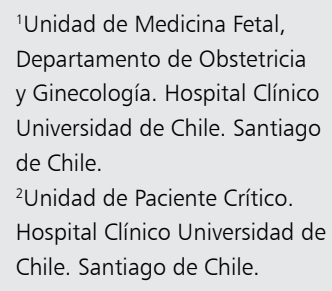

Recibido el 12 de octubre de 2014, aceptado el 13 de abril de 2015.

Correspondencia a:

Dr. Mauro Parra-Cordero. Unidad de Medicina Fetal. Hospital Clínico Universidad de Chile.

Santos Dumont 999, sector A primer piso. Independencia.

Santiago de Chile.

Teléfono: (2)29788881

mcparra@hcuch.cl
E l embarazo es un estado durante la vida de la mujer que conlleva una serie de adaptaciones fisiológicas con el fin de favorecer el desarrollo fetal (Tabla 1). La identificación de alteraciones en la función hepática durante el embarazo ha sido descrita en 3 a $5 \%$ de las gestantes ${ }^{1} \mathrm{e}$ ictericia, solamente en $0,1 \%{ }^{2}$, siendo gran parte de las alteraciones propias del embarazo o enfermedades hepáticas concomitantes con la gestación. Las hepatopatías propias del embarazo, a su vez, pueden estar relacionadas con preeclampsia (PE) (PE severa, síndrome de HELLP o hígado graso agudo del embarazo (HGAE)) o no relacionada con PE (hiperemesis gravídica o colestasia intrahepática del embarazo), siendo las relacionadas con $\mathrm{PE}$ las que se relacionan con una mayor severidad y mortalidad materna ${ }^{3}$.

Dentro de las hepatopatías concomitantes con el embarazo, éstas pueden ser alteraciones pre-existentes que se agudizan durante la gestación o aquellas que se manifiestan por primera vez durante el embarazo ${ }^{4}$. Las etiologías agudas de mayor riesgo son aquellas relacionadas con diversos tipos de hepatitis aguda, viral o medicamentos, y sus complicaciones (hepatitis aguda fulminante).

La siguiente revisión se centrará en aquellas afecciones hepáticas durante el embarazo que conllevan un riesgo vital materno, analizando la etiología y manejo de cada una de ellas.

\section{Hepatopatías agudas graves relacionadas con el embarazo}

Dentro de las patologías exclusivas del embarazo que tienen un potencial riesgo vital de la embarazada destacan: HGAE y PE severa/HE- 
Tabla 1. Adaptaciones fisiológicas de la función hepática durante el embarazo (adaptado de Hay)

\begin{tabular}{|ll|}
\hline Prueba & Rango de normalidad \\
Globulina & $\downarrow$ en $\gamma$-globulina, \\
& $\uparrow$ en $\alpha$ y $\beta$-globulina \\
Hemoglobina & $\downarrow$ a fines de tercer trimestre \\
\hline Bilirrubina & Sin cambios o levemente $\downarrow$ \\
\hline Transaminasas & Sin cambios \\
\hline Fosfatasas alcalinas & $\uparrow 2$ a 4 veces \\
\hline Tiempo protrombina & Sin cambios \\
\hline Fibrinógeno & $\uparrow 50 \%$ \\
\hline Ceruloplasmina & Incrementa \\
\hline Colesterol & $\uparrow 2$ veces \\
\hline Triglicéridos & Incrementa \\
\hline Rcto glóbulos blancos & Incrementa \\
\hline
\end{tabular}

LLP. Ambas entidades se caracterizan por afectar preferentemente en el tercer trimestre (3T) de la gestación.

\section{Hígado graso agudo del embarazo}

\section{Definición}

Enfermedad rara y potencialmente fatal, caracterizada por infiltración grasa microvesicular en los hepatocitos, casi exclusiva del $3 \mathrm{~T}$ de embarazo ${ }^{1}$, de carácter reversible post parto ${ }^{5}$. La prevalencia reportada en países anglosajones es de 1:7.000 a $1: 20.000^{6,7}$. En Chile, reportes demuestran tasas similares a las internacionales, con cifras de 1:15.900 recién nacidos vivos ${ }^{8}$. Su deterioro agudo se presenta no sólo como insuficiencia hepática, sino que también puede afectar otros órganos.

\section{Etiopatogenia}

Histológicamente, se describe una infiltración grasa microvesicular de los hepatocitos, los cuales además presentan signos de inflamación. Estos hallazgos predominan en las zonas hepáticas II y III (zonas pericentral y medias) ${ }^{7}$. Junto a esto se ha observado, con microscopía electrónica, la presencia de alteraciones inespecíficas en el tamaño y forma mitocondrial.

La causa del HGAE es desconocida, sin embargo, recientemente se ha demostrado una asociación con defectos congénitos de la beta-oxidación de los ácidos grasos, específicamente, un déficit de la 3-hydroxyacyl-CoA dehidrogenasa de cadena larga (LCHAD, por sus siglas en inglés) ${ }^{9}$. La LCHAD está encargada de catalizar el tercer paso de la beta-oxidación de los ácidos grasos en la mitocondria. Su forma homocigota se ha asociado a riesgo elevado de hígado graso agudo en el neonato, por lo que ante una madre con HGAE siempre es recomendable estudiar al recién nacido buscando dirigidamente este defecto ${ }^{7}$.

No está completamente definido el mecanismo de acción de la enfermedad, sin embargo, se ha planteado una acumulación de los metabolitos del LCHAD mitocondrial, los cuales tendrían un efecto tóxico en los hepatocitos maternos ${ }^{10}$. Estos pueden provenir tanto de la madre como del feto portador de la mutación, pudiendo demostrarse en placentas de pacientes con HGAE un aumento en las concentraciones de ácido araquidónico, lo que se ha asociado a hepatotoxicidad e inducción de apoptosis de los hepatocitos ${ }^{11}$.

\section{Cuadro clínico}

Se manifiesta durante el 3T, preferentemente entre las 32-36 semanas, pero con reportes incluso a las 38 semanas $^{5,12-14}$. Dentro de los síntomas más frecuentes se describen las náuseas y vómitos, malestar general, dolor abdominal, ictericia y fiebre. Menos frecuentemente, se observa prurito, hipertensión y cefalea. La presencia de encefalopatía hepática es muy infrecuente, pero es un cuadro grave de la enfermedad.

Las alteraciones séricas de función hepática (hiperbilirrubinemia, tiempo de protrombina aumentado, TTPA alterado, déficit de fibrinógeno), aumento de transaminasas e hipoglicemia marcada, son los hallazgos bioquímicos descritos con mayor frecuencia ${ }^{12,14}$. En cuadros severos puede observarse alteración de la función renal, hiperamonemia, trombocitopenia y coagulación intravascular diseminada (CID $)^{4,14}$. Ch'ng et $\mathrm{al}^{15}$ describieron los criterios diagnósticos para HGAE (Tabla 2), por medio del uso de al menos 6 criterios, aumentar la sensibilidad diagnóstica, siendo de utilidad en centros que no dispongan de biopsia hepática.

\section{Manejo}

Las alteraciones tanto clínicas como bioquímicas no mejoran en el período antenatal, y un 
Tabla 2. Criterios diagnósticos de Hígado Graso Agudo del embarazo (adaptado de referencia 15)

Seis o más de los siguientes, en ausencia de otra causa,
durante el tercer trimestre del embarazo:
Dolor abdominal
Vómitos
Poliuria/polidipsia
Encefalopatía
Leucocitosis
Ascitis
Coagulopatía
Hipoglicemia
Hiperbilirrubinemia
Hiperamonemia
Aumento de transaminasas
Disfunción renal aguda
Hiperuricemia
Grasa microvesicular en biopsia hepática*

* La presencia de infiltrado graso en la biopsia es criterio mayor para realizar el diagnóstico sin necesidad de cumplir con 5 criterios adicionales.

retardo en la interrupción del embarazo puede llevar a un mayor compromiso materno, por lo que ante una paciente con diagnóstico certero de HGAE se requiere interrupción del embarazo precoz ${ }^{7}$. La vía del parto debe ser decidida según condiciones obstétricas y grado de compromiso materno. Una revisión de 11 casos de HGAE demostró la utilidad del ultrasonido (US) en el estudio, siendo compatible con hígado graso en $81,8 \%$ de los casos. La tomografía computarizada (TAC) de abdomen demostró un rendimiento inferior al US ${ }^{16}$.

Una vez resuelto el embarazo, los síntomas maternos y las alteraciones bioquímicas progresan hacia la mejoría. Recientemente, Nelson et $\mathrm{al}^{6}$ evaluaron el resultado perinatal y el comportamiento de las alteraciones bioquímicas, una vez interrumpido el embarazo, en 51 pacientes, durante un período de 37 años en una institución norteamericana. La sobrevida al nacer fue de sólo $57 \%$, con $40 \%$ de partos prematuros. Las alteraciones maternas más observadas fueron coagulopatía, encefalopatía y pancreatitis. La mejoría clínica se objetivó alrededor del $3^{\circ}$ a $4^{\circ}$ día post parto, mientras que las alteraciones bioquímicas tienden a tardar más, demostrando una mejoría alrededor del $7^{\circ}$ día post parto.

Una alternativa médica a la interrupción del embarazo fue propuesta recientemente por Seyyed Majidi et $a{ }^{17}$. Los autores reportaron tres casos de pacientes con HGAE que fueron manejadas exitosamente con plasmaféresis, tratamiento que demostró proteger los hepatocitos a través de una disminución del estrés oxidativo que lleva a daño en la función mitocondrial. Estos resultados fueron corroborados por otros autores ${ }^{18,19}$. Sin embargo, existen grupos que no apoyan esta conducta, basándose en la evolución rápidamente favorable de la gran mayoría de las pacientes con HGAE manejadas exclusivamente con medidas de soporte habitual en Unidad de Cuidados Intensivos (UCI).

El estudio de la mutación del gen HDAHA debe ser realizado tanto en la paciente afectada por HGAE como en el neonato, estrategia que ha demostrado disminuir en forma considerable las complicaciones severas neonatales de niños afectados.

\section{Síndrome de HELLP}

\section{Definición}

Descrito por primera vez por Weinstein en 1982, acrónimo por sus iniciales en inglés, hemólisis (con presencia de esquistocitos característicos en la citología de sangre periférica), elevación de enzimas hepáticas y disminución de recuento plaquetario $^{20}$. Este cuadro se observa en $5-10 \%$ de las pacientes portadoras de PE. Sin embargo, su incidencia en la población general es muy baja, con valores cercanos a $0,6 \%$ de los embarazos ${ }^{4}$. Estas cifras concuerdan con valores locales. Parra et $\mathrm{a}^{21}$ demostraron que $4,9 \%$ de las pacientes con PE controladas en el Hospital Clínico de la Universidad de Chile cursaron con HELLP completo. La variante incompleta del HELLP ha sido descrita como la presencia de dos de los componentes de la triada diagnóstica, no existiendo consenso en diversos grupos de estudio en incluir esta presentación.

\section{Etiopatogenia}

El daño hepático descrito en el HELLP es atribuido al depósito de fibrina intravascular, 
Tabla 3. Diagnóstico diferencial entre síndrome de HELLP e Hígado Graso Agudo del Embarazo (adaptado de Kingham ${ }^{12}$ y Rahman ${ }^{14}$ )

\begin{tabular}{|ll|}
\hline Hígado graso agudo del embarazo & Síndrome de HELLP \\
\hline Aumento tiempo de protrombina & Tiempo de protrombina normal \\
\hline Transaminasas muy elevadas & Transaminasas moderadamente elevadas \\
\hline Bilirrubina elevada & Bilirrubina normal \\
\hline Hipoglicemia & Glicemia normal \\
\hline Hiperamonemia & Amonemia normal \\
\hline Trombocitopenia leve & Trombocitopenia marcada \\
\hline LDH normal & LDH elevada \\
\hline
\end{tabular}

hipovolemia e hipertensión sinusoidal que trae como consecuencia una elevación moderada de los niveles de bilirrubina y de las transaminasas ${ }^{4}$. La trombocitopenia es causada por el daño endotelial sistémico, que lleva a depósito de fibrina, junto con activación y consumo plaquetario ${ }^{13}$. La hemólisis es causada por daño directo de los hematíes en el endotelio disfuncional, produciendo una hemólisis microangiopática ${ }^{22}$, que ocasiona la elevación en los niveles de LDH séricos. La hemoglobina liberada en la sangre materna es transformada a bilirrubina no conjugada a nivel esplénico o se une a la haptoglobina para ser eliminada a nivel hepático, manifestándose clínicamente como una disminución de los niveles de hemoglobina en sangre materna ${ }^{23}$.

\section{Cuadro clínico}

Aunque se han descrito casos de HELLP desde las 20 semanas de gestación, lo más habitual es la presentación entre las 27 y 36 semanas, $10 \%$ antes de las 27 semanas $^{14}$ y hasta en $25 \%$ post parto $^{12}$. Uno de los síntomas más frecuentemente reportados es el dolor abdominal (36-86\%), que predomina en epigastrio e hipocondrio derecho. Las náuseas, vómitos y cefalea son descritas habitualmente, por lo que es fundamental hacer el diagnóstico diferencial con el HGAE (Tabla 3). A pesar de su alta asociación con $\mathrm{PE}$, la hipertensión y proteinuria pueden estar ausentes en $15 \%$ y $6 \%$, respectivamente $\mathrm{e}^{14}$.

La sensación de malestar general se observa habitualmente los días previos al diagnóstico de la enfermedad, pudiendo confundirse con cuadros gastrointestinales como reflujo gastroesofágico o

gastritis aguda. Formas más graves de presentación son observadas por su asociación con PE severa (encefalopatía hipertensiva, insuficiencia renal aguda, distrés respiratorio, etc.).

A pesar de los síntomas descritos (muy semejantes al HGAE y a la PE severa), el diagnóstico de HELLP se realiza según los criterios de Tennessee, descritos por Sibai en 1990 (Tabla 4) ${ }^{24}$.

Hasta la fecha se han descrito marcadores clínicos y bioquímicos como predictores de gravedad materna. Dentro de los parámetros clínicos destaca el dolor epigástrico, cefalea, alteraciones visuales y las náuseas. Cavkaytar et $\mathrm{al}^{25}$, en una revisión de 61 pacientes con HELLP, demostraron que estos marcadores fueron superiores a los marcadores bioquímicos en la predicción de complicaciones maternas graves. Los marcadores bioquímicos que se asocian más a complicaciones maternas son: $\mathrm{LDH}>1400 \mathrm{U} / \mathrm{l}, \mathrm{GOT}>150 \mathrm{U} / \mathrm{l}$, GPT $>100 \mathrm{U} / \mathrm{l}$ y ácido úrico $>7,8 \mathrm{mg} / \mathrm{dl}^{26}$.

El HELLP se asocia a complicaciones severas perinatales y maternas. Las complicaciones neonatales son principalmente secundarias a la

\section{Tabla 4. Criterios diagnósticos de síndrome de HELLP}

\section{HELLP completo \\ $\mathrm{LDH} \quad>600 \mathrm{U} / \mathrm{l}$ \\ Plaquetas $<100.000$ \\ GOT $>70 \mathrm{U} / \mathrm{l}$}

HELLP incompleto

Sólo una o dos de los mencionados previamente 
prematurez y a la concomitancia con PE severa, como la restricción de crecimiento fetal ${ }^{27}$, el desprendimiento placentario ${ }^{25}$ y muerte fetal ${ }^{28}$. Dentro de las complicaciones maternas descritas, una de las más graves es la ruptura espontánea de un hematoma subcapsular hepático, que afortunadamente se observa sólo en $2 \%$ de estas pacientes ${ }^{22}$. Las coagulopatías severas (CID) se han descrito en hasta $20 \%$ de las pacientes, edema pulmonar en $12 \%$, falla renal en $8 \%$ y eclampsia en $8 \%{ }^{12,29}$.

\section{Manejo}

Frente a una paciente afectada por un síndrome de HELLP, el manejo debe enfocarse en estabilizar a la madre y prevenir las complicaciones perinatales. La estabilización materna dependerá del manejo cauteloso de fluidos endovenosos, monitorización hepática y manejo adecuado de la trombocitopenia.

El manejo de los fluidos endovenosos es fundamental, dado el estado de disfunción endotelial, hipoalbuminemia y el hipertono vasomotor, aumentando el riesgo de edema pulmonar agudo $y$ ascitis en casos de un aporte vigoroso de volumen, mientras que un aporte insuficiente puede exacerbar un daño renal agudo pre-renal inicial ${ }^{14}$. El monitoreo multisistémico, así como el manejo multidisciplinario de estas pacientes, debe ser proporcionado en una Unidad de Pacientes Críticos (UPC). Reportes recientes han demostrado tasas de ingreso a UCI en pacientes con HELLP de hasta $64 \%$, con estadías en dichas unidades entre 1 a 10 días $^{30}$.

La monitorización hepática dependerá del grado de alteración de las pruebas hepáticas y de las características del dolor abdominal. Frente a la sospecha de compromiso hepático se debe evaluar con US abdominal, para descartar un hematoma subcapsular hepático ${ }^{13}$; sin embargo, la TAC de abdomen con contraste o la resonancia nuclear magnética (RNM) han demostrado ser superiores en la identificación de esta complicación ${ }^{14,31}$. La identificación de un hematoma subcapsular hepático intacto requiere un manejo conservador en una UPC posterior a una pronta interrupción del embarazo. Por otra parte, ante la sospecha de una ruptura espontánea de un hematoma hepático, el manejo debe ser agresivo, con laparotomía inmediata y packing ${ }^{12}$. Alternativas quirúrgicas efectivas ante el fracaso del packing son el parche de omento, la sutura directa del lecho hepático, resección parcial hepática y la embolización hepática por medio de radiología intervencional ${ }^{32,34,35}$. Ante el fracaso del manejo quirúrgico, debe considerarse el uso de derivados hemostáticos como medida de rescate ${ }^{35}$. El soporte posterior debe ser entregado en una UPC para manejo agresivo del shock hemorrágico ${ }^{36}$. La ruptura hepática, a pesar de ser una complicación muy infrecuente ( 1 cada 40.000 a 250.000 embarazos ${ }^{32}$, determina una elevada letalidad materna y fetal, siendo considerada por algunos autores como una indicación de trasplante hepático ${ }^{33}$.

El manejo de la trombocitopenia ha generado mucha controversia. Diversos estudios no aleatorizados describieron, hace 20 años, que el uso de dexametasona antenatal se asocia con una mejoría significativa del recuento plaquetario y de la sintomatología ${ }^{37,38}$. A pesar de estos resultados alentadores, estudios posteriores aleatorios, controlados con placebo, de Fonseca y Katz ${ }^{39,40}$, no pudieron demostrar este beneficio tanto antenatal como post parto (HR 1,2; IC 95\% 0,8-1,8 para el estudio de Fonseca y $p=0,841$ entre ambos grupos para estudio de Katz). Estos resultados fueron corroborados en un meta-análisis reciente de Cochrane, que comparó el uso de corticoides antenatales versus placebo o no tratamiento en 11 estudios de distribución aleatoria (IC 95\% $0,24-1,10)^{41}$. Sin embargo, en un subanálisis de los datos, la dexametasona demostró ser significativamente superior a la betametasona en mejorar los niveles plaquetarios, por lo que aún queda espacio para más estudio en esta área.

El momento de interrupción del parto va a depender de la edad gestacional y el compromiso sistémico materno. Sibai et $\mathrm{al}^{42}$ plantean que ante una paciente con PE severa asociada con un HELLP y $<34$ semanas, con un cuadro estable, la interrupción del embarazo debiera ser realizada posterior a las $48 \mathrm{~h}$ de corticoides para inducir madurez pulmonar fetal. Frente a cuadros graves o de progresión rápida, se requerirá una interrupción inmediata del embarazo. La vía de parto dependerá de condiciones obstétricas, prefiriendo la vía vaginal. A pesar de esta indicación teórica, en la práctica, ante cuadros graves con compromiso sistémico, la opción preferida es la vía alta, con tasas de cesárea reportada de hasta $68 \%{ }^{43}$. El manejo posterior al parto debe ser en una UPC.

Durante el control del puerperio en UPC debe evaluarse periódicamente la presencia de disfun- 
ción de órganos o sistemas por escalas validadas, como el puntaje SOFA ${ }^{44}$. Ante la presencia de disfunción de dos o más sistemas en pacientes con HELLP, una alternativa de manejo descrita es el uso de plasmaféresis. Eser et $\mathrm{al}^{45}$ evaluaron retrospectivamente a 29 pacientes consecutivas con diagnóstico de HELLP, en un período de tres años, que fueron manejadas precozmente con plasmaféresis, considerando como grupo control a 26 pacientes manejadas sin plasmaféresis durante los seis años previos. Los resultados obtenidos demostraron una disminución significativa de la mortalidad materna ( $0 \%$ vs $23,1 \% ; \mathrm{p}=0,006)$ y de la permanencia en UCI (5,86 días vs 10,5 días; $\mathrm{p}=0,0001$ ), asociada a una recuperación precoz de los parámetros de laboratorio.

\section{Hepatopatías agudas graves concomitantes con el embarazo}

En este grupo de patologías nos centraremos en las causas más frecuentes de hepatitis aguda fulminante: hepatitis aguda viral y por intoxicación medicamentosa. La diferenciación clínica con patologías hepáticas propias del embarazo (HGAE, HELLP) puede ser difícil basado sólo en la clínica, por lo que la anamnesis es una herramienta fundamental para definir la causa.

\section{Hepatitis fulminante (HF)}

\section{Definición}

Se considera un tipo de presentación severa de hepatitis aguda, potencialmente reversible, caracterizado por un inicio agudo, de progresión rápida, asociado a encefalopatía hepática de grado variable, complicaciones graves y pésimo pronóstico ${ }^{46}$. Luego del inicio agudo, la paciente evoluciona con ictericia marcada asociado a encefalopatía hepática dentro de las ocho semanas posteriores al inicio de la enfermedad, sin una patología hepática previa de base. La mortalidad descrita es de alrededor de $60-75 \%{ }^{46,47}$. Su asociación con embarazo se describe en menos de $0,1 \%{ }^{48}$. La descripción general de la HF en un contexto distinto al embarazo está fuera del alcance de esta revisión ${ }^{49}$.

\section{Etiopatogenia}

Las causas más frecuentemente descritas de HF son las infecciones virales y las reacciones adversas a medicamentos. Dentro de las infecciones virales destacan: virus hepatitis (A-E), virus herpes simple y citomegalovirus ${ }^{46}$.

La infección por virus hepatitis es la principal causa infecciosa de HF. En países subdesarrollados con altas tasas de prevalencia de infección, el VHE es la principal causa de HF durante el embara$\mathrm{zo}^{50-52}$. Sin embargo, en Chile no existen reportes oficiales de infección por VHE en la gestante, debiendo considerarse el perfil epidemiológico local al enfrentarse a una embarazada con sospecha de un agente infeccioso como causal de la HF.

Se desconoce la razón por la cual la gestante es más susceptible a una evolución tórpida por VHE, pero se ha planteado que podría basarse en una disminución de la respuesta inmune celular (Th-1), al parecer secundaria a los altos niveles de hormonas esteroideas que inducirían cambios inmunológicos, los cuales influirían sobre la expresión y replicación viral durante el embarazo ${ }^{53}$. En países desarrollados, los principales agentes infecciosos causales de HF son los VH A y B; sin embargo, se ha descrito que los VH E, A y herpes simple presentan una evolución más severa en la embarazada, especialmente si es adquirida durante el $3 \mathrm{~T}^{13}$, con tasas de mortalidad maternas y perinatales alrededor de $20 \%{ }^{54}$. A pesar de esto, sólo $1 \%$ de las pacientes afectadas por VHA evoluciona a una $\mathrm{HF}^{47}$. La evolución a HF por infección viral es desconocida, pero se ha planteado una respuesta inmune exagerada de las células hepáticas infectadas.

La HF por virus herpes simple es muy infrecuente, pero con una mortalidad en sujetos no tratados de más de $80 \%{ }^{55}$. En el embarazo ocurre principalmente en el 3T, pudiendo ser causada por los serotipos 1 y 2 .

Los medicamentos son otra causa importante de HF, dentro de los cuales la principal causa es el uso de paracetamol en dosis tóxicas ${ }^{56}$. El daño hepático producido por el consumo de paracetamol es dosis dependiente y se caracteriza por un daño agudo que presenta un patrón característico de necrosis hepatocelular pericentral. El paracetamol normalmente es metabolizado por las enzimas hepáticas glucuroniltransferasa y sulfotransferasa, produciendo metabolitos estables que se eliminan por la orina. En condiciones de consumo excesivo de este fármaco se produce una metabolización alterna mediada por estrés oxidativo (citocromo $\mathrm{P} 450$ ) que lleva a la producción del producto intermedio $\mathrm{N}$-acetil-benzoquinona imina (NAPQI). 
El NAPQI produce una unión covalente, mediada por estrés oxidativo, con las proteínas intracelulares de los hepatocitos que lleva a disfunción y necrosis hepatocelular. Junto a esto, una acumulación de NAPQI puede llevar a una depleción de los depósitos de glutatión intrahepático, con la consiguiente toxicidad hepática ${ }^{56}$.

\section{Cuadro clínico}

La HF viral puede manifestarse en cualquier trimestre del embarazo, pero predomina en el 3T. Los síntomas iniciales son vagos, con malestar general, náuseas, vómitos, hipodinamia, fiebre y anorexia. Posteriormente se asocia ictericia de escleras y cutánea, dolor abdominal, prurito cutáneo y sangrado de mucosas ${ }^{46}$. Una vez que el daño hepático progresa, se asocia compromiso neurológico, con los diversos grados de encefalopatía hepática conocidos, y complicaciones multisistémicas severas.

Desde el punto de vista de las alteraciones bioquímicas, los hallazgos más característicos son un incremento súbito de los niveles de bilirrubina (generalmente sobre 1,9 mg/dL), disminución del tiempo de protrombina (habitualmente $<40 \%$ ), aumento severo de las transaminasas e inversión de la relación albúmina/globulina ${ }^{46}$. El trastorno de la coagulación es generalmente severo.

Un estudio reciente estableció a través de un análisis de regresión logística que las variables clínicas más asociadas con predicción de mortalidad materna fueron el dolor abdominal (OR $5,68)$, oliguria (OR 14,09) y la presencia de ascitis (OR 5,17). De los parámetros bioquímicos, el de mayor asociación fue el valor de bilirrubina total $(\mathrm{OR} 1,17)^{57}$.
El cuadro clínico de HF asociado a consumo de paracetamol es muy semejante al cuadro de origen viral, por lo que la anamnesis es fundamental para identificar consumo de fármacos potencialmente causales.

\section{Manejo}

La determinación de serología viral para $\mathrm{VH}$ A-E, CMV y herpes simple 1 y 2 es parte imprescindible del manejo. Toda paciente diagnosticada con HF requiere de manejo intensivo con soporte vital en una UPC. Si el cuadro debuta en el 3T, se debe interrumpir el embarazo precozmente, ya que el pronóstico fetal es ominoso en pacientes que requieren trasplante hepático con embarazo in-situ $^{47,58,59}$, con tasas de sobrevida neonatal de tan sólo $27 \%{ }^{47}$. Sin embargo, reportes aislados han demostrado buen resultado perinatal en pacientes trasplantadas durante el embarazo ${ }^{52,60-62}$. Si el cuadro debuta en el segundo trimestre, se debe priorizar la necesidad de trasplante y monitorizar al feto en forma periódica, dada la necesidad de uso de drogas inmunosupresoras y exposición a radiación durante la monitorización post operatoria ${ }^{47}$.

En pacientes con intoxicación por paracetamol el uso de $\mathrm{N}$-acetilcisteína ha demostrado mejorar el daño hepático, si éste es administrado precozmente $^{56}$. A pesar de esto, la necesidad de trasplante no se verá favorecida por el uso de esta terapia ante una paciente que ya ha desarrollado HF por paracetamol.

El uso de los criterios pronósticos del King's College para evaluar la necesidad de trasplante hepático ha demostrado ser de utilidad en el manejo de la HF independiente de la causa (Tabla 5) ${ }^{63}$.

Tabla 5. Criterios del Hospital King's College para decisión de trasplante hepático en pacientes con falla hepática aguda (FHA)

\section{FHA asociada a paracetamol}

$\mathrm{pH}<7,3$ o

INR $>6,5$; creatininemia $>3,4 \mathrm{mg} / \mathrm{dl}$, y encefalopatía grado III o IV

\section{FHA no asociada a paracetamol}

INR $>6,5$ o

Tres de los siguientes criterios, independiente del grado de encefalopatía:

- Edad < 10 o $>40$ años

- Hepatitis noA noB o reacción medicamentosa idiosincrática

-Duración de ictericia previo a la encefalopatía > 7 días

- INR > 3,5

Bilirrubinemia $>17,5 \mathrm{mg} / \mathrm{dl}$ 
Finalmente, estudios recientes han evaluado nuevas terapias para pacientes con daño hepático agudo que plantean resultados promisorios. Cao et $\mathrm{al}^{64}$ demostraron en modelos animales que el uso de células madre con diferenciación hepática inducida, tanto in vitro como in vivo, mejoraron la función hepática, prolongando la sobrevida de los animales afectados por daño hepático agudo experimental.

\section{Conclusión}

El daño hepático agudo es una entidad grave durante el embarazo que requiere un manejo en unidades de alta complejidad. En nuestro establecimiento, en un período de 5 años, 89 pacientes requirieron manejo en UPC y 24 correspondieron a patología hepática aguda relacionada con el embarazo (23 HELLP y un HGAE). Dentro de las pacientes trasladadas a UPC por PE severa o sus complicaciones, el HELLP correspondió a $55 \%$ de los casos. A pesar de la gravedad de estas pacientes, el manejo precoz y multidisciplinario permitió alcanzar $100 \%$ de sobrevida materna ${ }^{65}$.

En conclusión, el enfoque de una paciente con sospecha de daño hepático agudo requiere una anamnesis rigurosa y evaluación dirigida para identificar las causas más graves presentadas en esta revisión. El manejo multidisciplinario es fundamental para lograr un buen resultado materno-fetal.

\section{Referencias}

1. Hay J. Liver Disease in Pregnancy. Hepatology 2008; 47: 1067-76.

2. Than NN, Neuberger J. Liver abnormalities in pregnancy. Best Pract Res Clin Gastroenterol 2013; 27 (4): 565-75.

3. Curiel-Balsera E, Prieto-Palomino MA, Muñoz-Bono J, Ruiz de Elvira MJ, Galeas JL, Quesada García G. Análisis de la morbimortalidad materna de las pacientes con preeclampsia grave, eclampsia y síndrome HELLP que ingresan en una Unidad de Cuidados Intensivos gineco-obstétrica. Med Intensiva 2011; 35 (8): 478-83.

4. Joshi D, James A, Quaglia A, Westbrook R, Heneghan M. Liver disease in pregnancy. Lancet 2010; 375: 594605.

5. Duarte J, Díaz S, Eng V, Castro J, Velásquez V. Hígado graso agudo del embarazo y defectos de la betaoxida- ción. Med Int Mex 2007; 23 (5): 424-35.

6. Nelson DB, Yost NP, Cunningham FG. Acute fatty liver of pregnancy: clinical outcomes and expected duration of recovery. Am J Obstet Gynecol 2013; 209 (5): 456. e1-456.e7.

7. Bacq Y. Liver diseases unique to pregnancy: A 2010 update. Clin Res Hepatol Gastroenterol 2011; 35: 182-93.

8. Reyes H, Sandoval L, Wainstein A, Ribalta J, Donoso S, Smok G, et al. Acute fatty liver of pregnancy: a clinical study of 12 episodes in 11 patients. Gut 1994; 35: 101-6.

9. Wilcken B, Leung KC, Hammond J, Kamath R, Leonard JV. Pregnancy and fetal long-chain 3-hydroxyacyl coenzyme A dehydrogenase deficiency. Lancet 1993; 341: 407-8.

10. Rector RS, Payne RM, Ibdah JA. Mitochondrial trifunctional protein defects: clinical implications and therapeutic approaches. Adv Drug Deliv Rev 2008; 60: 1488-96.

11. Natarajan SK, Thangaraj KR, Eapen CE, Ramachandran A, Mukhopadhya A, Mathai M, et al. Liver injury in acute fatty liver of pregnancy: possible link to placental mitocondrial dysfunction and oxidative stress. Hepatology 2010; 51: 191-200.

12. Kingham JGC. Liver Disease in Pregnancy. Clin Med 2006; 6: 34-40.

13. Boregowda G, Shehata HA. Gastrointestinal and Liver Disease in Pregnancy. Best Pract Res Clin Obstet Gynaecol 2013; 27: 835-53.

14. Rahman TM, Wendon J. Severe hepatic dysfunction in pregnancy. Q J Med 2002; 95: 343-57.

15. Ch'ng CL, Morgan M, Hainsworth I, Kingham JG. Prospective study of liver dysfunction in pregnancy in Southwest Wales. Gut 2002; 51 (6): 876-80.

16. Wei Q, Zhang L, Liu X. Clinical diagnosis and treatment of acute fatty liver of pregnancy: A literature review and 11 new cases. J Obstet Gynaecol Res 2010; 36 (4): 75156.

17. Seyyed Majidi MR, Vafaeimanesh J. Plasmapheresis in acute Fatty liver of pregnancy: an effective treatment. Case Rep Obstet Gynecol 2013; doi: 10.1155/2013/615975.

18. Jin F, Cao M, Bai Y, Zhang Y, Yang Y, Zhang B. Therapeutic effects of plasma exchange for the treatment of 39 patients with acute fatty liver of pregnancy. Discov Med 2012; 13: 369-73.

19. Martin JN Jr, Briery CM, Rose CH, Owens MT, Bofill JA, Files JC. Postpartum plasma exchange as adjunctive therapy for severe acute fatty liver of pregnancy. J Clin Apheresis 2008; 23: 138-43.

20. Weinstein L. Syndrome of hemolysis, elevated liver enzymes, and low platelet count: a severe consequence of 
hypertension in pregnancy. Am J Obstet Gynecol 1982; 142: 159-67.

21. Parra M, San Martín A, Valdés E, Hasbun J, Quiroz L, Shepeler M, et al. Espectro clínico de la preeclampsia: estudio comparativo de sus diversos grados de severidad. Rev Chil Obstet Ginecol 2007; 72 (3): 169-75.

22. Haram K, Svendsen E, Abildgaard U. The HELLP syndrome: Clinical issues and management. A Review. BMC Pregnancy Childbirth 2009; 9: 8. doi: 10.1186/1471-2393-9-8.

23. Wilke G, Rath W, Schutz E, Armstrong VW, Kuhn W. Haptoglobin as a sensitive marker of hemolysis in HELLP-syndrome. Int J Gynaecol Obstet 1992; 39: 29-34.

24. Sibai BM. The HELLP syndrome (hemolysis, elevated liver enzymes, and low platelets): much ado about nothing? Am J Obstet Gynecol 1990; 162: 311-6.

25. Cavkaytar S, Ugurlu EN, Karaer A, Tapisiz OL, Danisman N. Are clinical symptoms more predictive than laboratory parameters for adverse maternal outcome in HELLP syndrome? Acta Obstet Gynecol Scand 2007; 86: 648-51.

26. Magann EF, Martin JN Jr. Twelve steps to optimal management of HELLP syndrome. Clin Obstet Gynecol 1999; 42: 532-50.

27. Aslan H, Gul A, Cebeci A. Neonatal outcome in pregnancies after preterm delivery for HELLP syndrome. Gynecol Obstet Invest 2004; 58: 96-9.

28. Gul A, Cebeci A, Aslan H, Polat I, Ozdemir A, Ceylan Y. Perinatal outcomes in severe preeclampsia-eclampsia with and without HELLP syndrome. Gynecol Obstet Invest 2005; 59: 113-8.

29. Sibai BM, Ramadan MK, Usta I, Salama M, Mercer BM, Friedman SA. Maternal morbidity and mortality in 442 pregnancies with hemolysis, elevated liver enzymes, and low platelets (HELLP syndrome). Am J Obstet Gynecol 1993; 169: 1000-6.

30. Fitzpatrick KE, Hinshaw K, Kurinczuk JJ, Knight M. Risk Factors, Management, and Outcomes of Hemolysis, Elevated Liver Enzymes, and Low Platelets Syndrome and Elevated Liver Enzymes, Low Platelets Syndrome. Obstet Gynecol 2014; 123: 618-27.

31. Barton JR, Sibai BM. Hepatic imaging in HELLP síndrome (hemolysis, elevated liver enzymes, and low platelet count). Am J Obstet Gynecol 1996; 174: 1820-5.

32. Wicke C, Pereira PL, Neeser E, Flesch I, Rodegerdts EA, Becker HD. Subcapsular liver hematoma in HELLP syndrome: Evaluation of diagnostic and therapeutic options-a unicenter study. Am J Obstet Gynecol 2004; 190 (1): 106-12.

33. Pereira SP, O’Donohue J, Wendon J, Williams R. Maternal and perinatal outcome in severe pregnancy-related liver disease. Hepatology 1997; 26: 1258-62.

34. Araujo AC, Leao MD, Nobrega MH, Bezerra PF, Pereira FV, Dantas EM, et al. Characteristics and treatment of hepatic rupture caused by HELLP syndrome. Am J Obstet Gynecol 2006; 195 (1): 129-33.

35. Mihu D, Costin N, Mihu CM, Seicean A, Ciortea R. HELLP syndrome-a multisystemic disorder. J Gastrointestin Liver Dis 2007; 16 (4): 419-24.

36. Hunt BJ. Bleeding and Coagulopathies in Critical Care. N Engl J Med 2014; 370: 847-59.

37. Magann EF, Perry KG, Meydrech EF, Harris RL, Chauchan SP, Martin JN. Postpartum corticosteroids: accelerated recovery from the syndrome of hemolysis, elevated liver enzymes and low platelets (HELLP). Am J Obstet Gynecol 1994; 171: 1154-8.

38. Vigil-De Gracia P, García-Cáceres E. Dexamethasone in the post-partum treatment of HELLP syndrome. Int J Gynecol Obstet 1997; 59: 217-21.

39. Fonseca J, Méndez F, Cataño C, Arias F. Dexamethasone treatment does not improve the outcome of women with HELLP syndrome: A double-blind, placebo-controlled, randomized clinical trial. Am J Obstet Gynecol 2005; 193: 1591-8.

40. Katz L, de Amorim MMR, Figueiroa JN, Pinto e Silva JL. Postpartum dexamethasone for women with hemolysis, elevated liver enzymes, and low platelets (HELLP) syndrome: a double-blind, placebo-controlled, randomized clinical trial. Am J Obstet Gynecol 2008; 198: 283.e1283.e8.

41. Woudstra DM, Chandra S, Hofmeyr GJ, Dowswell T. Corticosteroids for HELLP (hemolysis, elevated liver enzymes, low platelets) syndrome in pregnancy. Cochrane Database Syst Rev 2010 Sep 8; (9): CD008148. doi: 10.1002/ 14651858.CD008148.pub2.

42. Sibai BM, Barton JR. Expectant management of severe preeclampsia remote from term: patient selection, treatment, and delivery indications. Am J Obstet Gynecol 2007; 196: 514.e1-514.e9.

43. Martin-JN J, Perry KG Jr, Roberts WE. Plasma exchange for preeclampsia: III. Immediate peripartal utilization for selected patients with HELLP syndrome. J Clin Apheresis 1994; 9: 162-5.

44. Ferreira FL, Bota DP, Bross A, Mélot C, Vincent JL. Serial evaluation of the SOFA score to predict outcome in critically ill patients. JAMA 2001; 286 (14): 1754-8.

45. Eser B, Guven M, Unal A, Coskun R, Altuntas F, Sungur $\mathrm{M}$. The Role of Plasma Exchange in HELLP Syndrome. Clin Appl Thrombosis/Hemostasis 2005; 11 (2): 211-7.

46. Li XM, Ma L, Yang YB, Shi ZJ, Zhou SS. Clinical characteristics of fulminant hepatitis in pregnancy. World J Gastroenterol 2005; 11 (29): 4600-3. 
47. Simsek Y, Isik B, Karaer A, Celik O, Kutlu R, Aydin NE, et al. Fulminant hepatitis A infection in second trimester of pregnancy requiring living-donor liver transplantation. J Obstet Gynaecol Res 2012; 38 (4): 745-8.

48. Paternoster DM, Gerace PF, Manganelli F, Boccagni P, Cillo U, D'Amico D. Acute hepatic failure in pregnancy. Eur J Obstet Gynecol Reprod Biol 2004; 112: 230-2.

49. Bernal W, Wendon J. Acute Liver Failure. N Engl J Med 2013; 369: 2525-34.

50. Khuroo M.S, Kamili S. Aetiology, clinical course and outcome of sporadic acute viral hepatitis in pregnancy. J Viral Hepatitis 2003; 10: 61-9.

51. Mehta S, Singla A, Rajaram S. Prognostic factors for fulminant viral hepatitis in pregnancy. Int J Gynaecol Obstet 2012; 118: 172-5.

52. Shinde N, Patil T, Deshpande A, Gulhane R, Patil M, Bansod Y. Clinical profile, maternal and fetal outcomes of acute hepatitis e in pregnancy. Ann Med Health Sci Res 2014; 4 (suppl 2): S133-S9.

53. Jilani N, Das BC, Husain SA, Baweja UK, Chattopadhya D, Gupta RK, et al. Hepatitis E virus infection and fulminant hepatic failure during pregnancy. J Gastroenterol Hepatol 2007; 22 (5): 676-82.

54. Sultana R, Humayun S. Fetomaternal outcome in acute hepatitis E. J Coll Physicians Surg Pak 2014; 24 (2): 12730.

55. Thurman RH, König K, Watkins A, Weerasiri T, Permezel M. Fulminant herpes simplex virus hepatic failure in pregnancy requiring liver transplantation. Aust N Z J Obstet Gynaecol 2010; 50 (5): 492-4.

56. Fontana RJ. Acute liver failure including acetaminophen overdose. Med Clin North Am 2008; 92: 761-94.

57. Devarbhavi H, Kremers WK, Dierkhising R, Padmanabhan L. Pregnancy-associated acute liver disease and acute viral hepatitis: Differentiation, course and outcome. J Hepatol 2008; 49: 930-5.
58. Franko KR, Mekeel KL, Woelkers D, Khanna A, Hemming AW. Accidental Acetaminophen Overdose Results in Liver Transplant During Second Trimester of Pregnancy: A Case Report. Transplant Proc 2013; 45 (5): 2063-5.

59. Horikoshi Y, Itoh H, Kikuchi S, Uchida T, Suzuki K, Sugihara K, et al. Successful living donor liver transplantation for fulminant hepatic failure that manifested immediately after cesarean delivery. ASAIO J 2012; 58 (2): 174-6.

60. Jarufe N, Soza A, Pérez-Ayuso RM, Poblete JA, González $\mathrm{R}$, Guajardo $\mathrm{M}$, et al. Successful liver transplantation and delivery in a woman with fulminant hepatic failure occurring during the second trimester of pregnancy. Liver International 2006; 26: 494-7.

61. Maddukuri VC, Stephenson CD, Eskind L, Ahrens WA, Purdum P, Russo MW. Liver transplantation for acute liver failure at 11-week gestation with successful maternal and fetal outcome. Case Rep Transplant 2012; 2012:484080. doi: 10.1155/2012/484080.

62. Kimmich N, Dutkowski P, Krähenmann F, Müllhaupt B, Zimmermann R, Ochsenbein-Kölble N. Liver Transplantation during Pregnancy for Acute Liver Failure due to HBV Infection: A Case Report. Case Rep Obstet Gynecol 2013; 2013: 356560. doi: 10.1155/2013/356560.

63. O'Grady JG, Schalm SW, Williams R. Acute liver failure: redefining the syndromes. Lancet 1993; 342 (8866): 2735.

64. Cao H, Yang J, Yu J, Pan Q, Li J, Zhou P, et al. Therapeutic potential of transplanted placental mesenchymal stem cells in treating Chinese miniature pigs with acute liver failure. BMC Med 2012; 10: 56. doi: 10.1186/17417015-10-56.

65. Hasbun J, Sepúlveda-Martínez A, Cornejo R, Romero C. Morbilidad materna grave e ingreso a cuidado intensivo. Hospital Clínico Universidad de Chile (2006-2010). Rev Med Chile 2013; 141: 1512-9. 